\title{
A Call to Improve the Visibility and Access of the American College of Radiology Practice Parameters in Neuroradiology: A Powerful Value Stream Enhancer for Both Neuroradiologists and Patients
}

\author{
(D).E. Jordan and (D) A. Norbash
}

\begin{abstract}
SUMMARY: Clinical practice guidelines and clinical practice parameters are among the tools that clinicians and radiologists use to inform decision making in the diagnosis and treatment of patients. Radiologists have been urged to objectively establish their value and measurable contributions to patient care. Radiology's contribution to the health care value stream can be established in the development of sound clinical practice guidelines. Neuroradiologists have been quite active in developing clinical guidelines, particularly in collaboration with the American College of Radiology, but there is a need to increase the visibility and accessibility of such documents. Increasing access and visibility can contribute to improved patient outcomes and an improved overall quality of care.
\end{abstract}

ABBREVIATIONS: $A C R=$ American College of Radiology; AHCPR = Agency for Health Care Policy and Research; CPG = Clinical Practice Guideline; GIN = Guidelines International Network; IOM = Institute of Medicine; PP = Practice Parameter

Clinical Practice Guidelines (CPGs), clinical standards, consensus statements, and Practice Parameters are among the tools that clinicians use to inform clinical decision-making in the diagnosis and treatment of patients. ${ }^{1-3}$ When guidelines are rigorously developed using an evidence-based approach derived from scientific knowledge, clinical trials, expert opinion, and expert consensus, the result is advanced and improved patient outcomes and an improved overall quality of care. Clinical Practice Guidelines have become increasingly visible within medical specialties. There are currently over 3700 guidelines contained within the Guidelines International Network (GIN; https://www. g-i-n.net/) data base from 39 countries. ${ }^{1}$ Such guidelines are intended to help with routine and complex patient management decisions while reducing the variability of practice. Evidencebased guidelines inform health and public policy decisions and also guide appropriate allocations of resources. Concerning radiology, the terminology referring to this effort to minimize unnecessary practice variability has evolved from "standards," to "guidelines," to the currently favored terminology "Practice Parameters" (PPs). We will therefore refer to CPGs when the larger house of medicine is concerned, and when possible, we will refer

Received August 18, 2018; accepted after revision October 29.

From the Department of Radiology (J.E.J.), Providence Little Company of Mary Medical Center, Torrance, California; Department of Radiology (J.E.J.), Stanford University School of Medicine, Stanford, California; and Department of Radiology (A.N.), University of California, San Diego School of Medicine, La Jolla, California. Please address correspondence to John E. Jordan, MD, MPP, PO Box 6481, San Pedro, CA 90734; e-mail: jjordan_us@yahoo.com

http://dx.doi.org/10.3174/ajnr.A5923 to these Practice Guidelines as PPs when contemporary radiology is concerned. ${ }^{3}$

Neuroradiologists have actively collaborated with the American College of Radiology (ACR) in developing clinical Practice Parameters with the assistance of the ACR Commission on Neuroradiology; the ACR Committee on Practice Parameters, Neuroradiology; and the American Society of Neuroradiology Standards and Guidelines Committee. Neuroradiology subspecialty input and representation have also been voluntarily provided by additional national neuroradiology and subspecialty societies, including the American Society of Pediatric Neuroradiology, the American Society of Spine Radiology, the American Society of Functional Neuroradiology, the Society of Neurointerventional Surgery, and the ACR Committee on Practice Parameters and Technical Standards-Nuclear Medicine and Molecular Imaging.

\section{Background of CPG Development in the United States}

The Institute of Medicine (IOM) is a nonprofit organization within the National Academies of Sciences, Engineering, and Medicine. The IOM has defined Clinical Practice Guidelines, initially as a result of the Omnibus Budget Reconciliation Act of 1989. ${ }^{1,4}$ The Omnibus Act sought to address concerns regarding variability in medical practice, in addition to addressing uncontained health care costs. Among the principal objectives of The Omnibus Act was the creation of the Agency for Health Care Policy and Research (AHCPR). The AHCPR contracted with the IOM in 1990 to create the Committee to Advise the Public Health Service on Clinical Practice Guidelines. This committee then issued its report, Clinical Practice Guidelines: Directions for a New Program in 1990, and defined CPGs as "systematically developed 
Validity: Practice guidelines are valid if, when followed, they lead to the health and cost outcomes projected for them, with other things
being equal. A prospective assessment of validity will consider the projected health outcomes and costs of alternative courses of action,
the relationship between the evidence and recommendations, the substance and quality of the scientific and clinical evidence cited, and
the means used to evaluate the evidence.
Reliability/Reproducibility: Practice guidelines are reliable and reproducible. 1) if_-given the same evidence and methods for guidelines
development-another set of experts would produce essentially the same statements; and 2) if_-given the same circumstances—-the
guidelines are interpreted and applied consistently by practitioners or other appropriate parties. A prospective assessment of reliability
may consider the results of independent external reviews and pretests of guidelines.
Clinical Applicability: Practice guidelines should be as inclusive of appropriately defined patient populations as scientific and clinical
evidence and expert judgment permit, and they should explicitly state the populations to which statements apply.
Clinical Flexibility: Practice guidelines should identify the specifically known or generally expected exceptions to their recommendations.
Clarity: Practice guidelines should use unambiguous language, define terms precisely, and use logical, easy-to-follow modes of
presentation.
Multidisciplinary Process: Practice guidelines should be developed by a process that includes participation by representatives of key
affected groups. Participation may include serving on panels that develop guidelines, providing evidence and viewpoints to the panels,
and reviewing draft guidelines.
Scheduled Review: Practice guidelines should include statements about when they should be reviewed to determine whether revisions
are warranted, given new clinical evidence or changing professional consensus.
Documentation: The procedures followed in developing guidelines, the participants involved, the evidence used, the assumptions and
rationales accepted, and the analytic methods employed should be meticulously documented and described.

${ }^{a}$ Reproduced with permission from Graham et al.'

statements to assist practitioner and patient decisions about appropriate health care for specific clinical circumstances." ${ }^{1}$ The reputation of the AHCPR later was questioned as it undertook development and implementation of Clinical Practice Guidelines, ultimately resulting in changes in the name and charge of the AHCPR. Following congressional lobbying by physician groups under the Healthcare Research and Quality Act of 1999, the AHCPR became the Agency for Healthcare Research and Quality. The Agency for Healthcare Research and Quality focuses on outcomes and effectiveness research. ${ }^{5}$

There was considerable dissatisfaction and questionable quality concerning CPGs despite these developments. This dissatisfaction led Congress to authorize the United States Department of Health and Human Services to contract with the IOM to develop standards for Clinical Practice Guidelines. It was determined that these standards could be realized through the Medicare Improvements for Patients and Providers Act of 2008. ${ }^{6}$ The IOM then created the Committee on Standards for Developing Trustworthy Clinical Practice Guidelines to undertake this effort. This committee reconfigured both the definitions and recommendations of the IOM concerning CPGs. In its report, Clinical Practice Guidelines We Can Trust, ${ }^{1}$ the IOM defined CPGs in the following manner: "Clinical Practice Guidelines are statements that include recommendations intended to optimize patient care that are informed by a systematic review of evidence and an assessment of the benefits and harms of alternative care options." The IOM CPG report recommended 8 attributes that CPGs should have, including validity, reproducibility, clinical relevance, clinical flexibility, clarity, multidisciplinary process, scheduled review, and meticulous documentation (Table). ${ }^{1}$ The IOM also recognized the added value of expert consensus documents, clinical standards, white papers, and practice parameters, though it did not specifically assess them due to the limited scope of its mandate. Radiologists have collaborated with other specialties in developing CPGs and these other types of documents, though more collaboration is needed between radiologists and multidisciplinary groups to enhance the impact and visibility of radiology-related CPGs. ${ }^{7}$

\section{Historical Practice Parameter Development in Radiology}

As a result of the revised IOM definition of Clinical Practice Guidelines published in 2011, the ACR soon after renamed its CPGs-formerly known as the ACR Practice Guidelines and Technical Standards - the ACR Practice Parameters and Technical Standards. This specific change in verbiage from practice guidelines to Practice Parameters recognized that radiology differs from other clinical specialties, with an understanding that patient outcomes from radiology are challenging to assess. The change in verbiage was intended to convey the reality that PPs are collaborative documents and that collaboration in generating the PPs by necessity principally took place among radiology or imaging subspecialties, whereas cross-specialty nonimaging multidisciplinary collaboration was more limited. To better understand the nuances of deliberations that led to these changes, some background information of how the ACR PPs evolved may be helpful.

The 1980s witnessed accelerating medical costs and increasing calls by medical specialties, payers, and policy makers for the development of clinical guidelines. The variability of practice in health care and escalating costs alarmed many, and ultimately culminated in legislation to facilitate clinical guideline development. ${ }^{4}$ During this same period, the ACR took the initiative to develop clinical guidelines for radiologists, resulting in approval of its first 9 guidelines in 1990, referred to at the time as "standards." These standards proliferated to include subspecialties such as neuroradiology, and by 2003 , about 121 ACR standards had been developed. ${ }^{8}$ In May 2003, at the ACR Council Annual Meeting, the term "practice guidelines" was adopted as a preferred replacement for the term "standards," which would later be replaced by the term "Practice Parameters." The reason the term "practice guidelines" replaced standards in 2003 was the perception of greater flexibility for the practicing radiologist. The word "standards" was believed to confer less flexibility, requiring radiologists to practice in accordance with the standard or be judged as practicing substandard care. For a small number of the documents, the term "technical standard" was chosen when addressing subjects requiring strict adherence such as the development of 
equipment specifications, radiation safety issues, and instrument monitoring, and deviations would be considered contributing to substandard practice. ${ }^{8}$ Many of the technical standards were crafted and sponsored by the American Association of Physicists in Medicine.

ACR Practice Parameters are developed and refined through a lengthy process of gathering evidence and generating expert consensus. ${ }^{9}$ The PPs undergo additional scrutiny during committee member reviews, then subspecialty society review, and next general member review and commentary. Finally, PPs are debated, amended, revised, and approved or rejected by the ACR Council at its annual meeting. Once adopted by the Council, the proffered PP is finally considered a policy statement of the ACR. Rather than representing rigid guidelines, the PPs are designed to provide guidance and principles to improve the practice of radiology and health care outcomes. The technical standards, in contrast, serve the radiologists' needs while also providing guidance for physicians and medical physicists working in radiology and imaging. Other PPs are developed for the practice of radiation oncology.

As stated in the ACR Practice Parameters handbook, the Practice Parameters, "describe a range of acceptable approaches for the diagnosis and/or treatment of disease for most patients in most circumstances. Given differences in training, experience, and local conditions, the ACR Practice Parameters and technical standards acknowledge the need for health care providers to exercise their independent medical judgment in making decisions regarding the use and specific details of any procedure.... Used in conjunction with the ACR Appropriateness Criteria, it is expected that the ACR Practice Parameters and technical standards will increase the likelihood that appropriate procedures will be performed in a safe and acceptable manner and will help reduce unnecessary ones. ACR Practice Parameters and technical standards are intended to be living documents that are regularly reviewed and revised to reflect changes in radiologic and radiation oncology practice."

There are currently 175 ACR PPs and technical standards. Of these, 19 or nearly $11 \%$ have been primarily and individually developed by neuroradiologists, though 53 are relevant to the practice of neuroradiology, even though not developed by neuroradiologists. These relevant PPs range from such subjects as communication standards to PPs addressing approaches in the interpretation of various specific imaging modalities, with particular relevant PPs relating to interventional techniques. Other PPs relevant to the practice of neuroradiology include sonographic evaluation of the head/neck/brain/spine, vascular imaging, biopsy/ drainage procedures, nuclear medicine of the CNS (eg, PET, dementia), and radiography. ${ }^{10}$ A number of collaborative societies have participated in generating neuroradiology documents pertaining to the development of the PPs; these collaborating societies include the Society of Interventional Radiology, Society of Nuclear Medicine and Molecular Imaging, Society of Pediatric Radiology, American Association of Physicists in Medicine, ACR Committee on Body Imaging (Musculoskeletal), and the aforementioned neuroradiology subspecialty societies. Regarding technical standards, approximately 18 exist and are primarily established for equipment quality control for the principal use of medical physicists. ${ }^{10}$ Many of these PPs and standards documents are not readily familiar to the American Society of Neuroradiology or neuroradiology community members because they may be categorized under various modalities, specialties, or organ systems. Nevertheless, these relevant PPs and technical standards are meaningful and relevant for the house of neuroradiology.

\section{Challenges to Quality Practice Parameter Development}

High-quality evidence may be lacking in some disciplines, including radiology, challenging the development of bona fide evidencebased clinical guidelines. ${ }^{7}$ Much of the radiology literature deals with observational and anecdotal evidence, limiting the ability of radiology to establish the impact and effectiveness of radiologic services - especially diagnostic procedures - on downstream patient outcomes. Neuroradiology has a significant impact on social consequences, including costs of care and quality of life when considering such varied and ubiquitous neurologic diseases as stroke, dementia, and movement disorders, with the potential to guide patient management decisions and thereby improve outcomes. $^{11}$

Most medical specialties have succeeded in producing CPGs. Not all CPGs are based on appropriately high-quality evidence. The limited number of studies demonstrating the positive contribution of radiology to patient outcomes is problematic for all radiologists because patient outcomes are one of the few principal determinants of value in health care. Value in health care, as in other disciplines, should ideally be defined in reference to the customer who also happens to be the patient. Patient outcomes are therefore a central measure of value in health care from a population health and societal perspective, underlining the need for radiologists to understand and appreciate the actual outcomes of our patients. ${ }^{12}$

Incentives and payments are continuously evolving to include value-based credit, as payment gradually shifts from fee for service to value-based care. With this new calculus, the metrics that permit accurate measurement of both quality of delivered care and patient outcomes become increasingly important. An inherent limitation of radiology is that most of our published quality metrics are related more to structure and process than outcomes. For example, Narayan et $\mathrm{al}^{13}$ reported that a systematic review of the literature for quality metrics in radiology found measures related to outcomes in only $27 \%$, whereas the inverse, $73 \%$ of metrics, was related to structure and process. As outcomes are progressively studied with increasing sophistication in all health care domains, it is apparent that relatively few studies in other areas of health care have consistently and convincingly shown that adherence to CPGs improves patient outcomes. ${ }^{14,15}$ While it is reasonable to expect a positive correlation between these 2, more investigation in establishing this connection may be necessary if clinicians are to be fully convinced of the value of CPGs and if they are expected to incorporate them into their everyday practice.

\section{Why This Matters to the House of Neuroradiology}

Despite a limited number of high-quality studies, there is a growing body of evidence that CPGs enhance the value of care delivery by enhancing quality, improving clinical outcomes, and reducing costs. As 1 example, a report by the Agency for Healthcare Research and Quality found that an estimated 87,000 fewer deaths 
were associated with advances in addressing hospital-acquired conditions when comparing 2010 and 2014, with an estimated cost savings of $\$ 19.8$ billion. While the exact causes of the reductions were difficult to pinpoint, greater adoption and use of CPGs was thought to be a major contributing factor to this change. ${ }^{3,16}$

Radiologists have been advised-both within and outside the house of neuroradiology-to objectively establish their value and measurable contributions to patient care. The contribution of radiology to the health care value stream can be established in the development of sound clinical Practice Parameters. The PPs generally are not published in medical journals and, despite their high quality, are not readily accessed or used by potential users when one assesses the distribution of this knowledge by examining MEDLINE or PubMed searches. As disclosed by the National Library of Medicine, "Approximately 30,000 records are included in the PubMed journal list which is updated daily and includes all MEDLINE titles as well as other non-MEDLINE titles in PubMed.... The non-MEDLINE journals include those whose content is deposited in PubMed Central. PubMed Central is also the repository for author manuscripts that are required under the National Institutes of Health Public Access Policy to be deposited when the research is funded by National Institutes of Health.... National Library of Medicine creates a cataloging record for every journal with a full text article in PubMed Central. Therefore, while most MEDLINE journals over the years have citations for all substantive content published, some of the non-MEDLINE journals may contribute far fewer citations over the years." ${ }^{17}$ In this context, the ACR PPs continue to miss salient opportunities for vast exposure and visibility.

\section{Radiologists Accessing Practice Parameters}

Society will likely expect that radiologists establish and substantiate our contributions to improved patient outcomes. Neuroradiologists should be leaders in developing high-quality evidencebased clinical Practice Parameters to advance the progress of the specialty and improve patient care.

New Practice Parameters will continue to be developed in neuroradiology and radiology, and it is essential that these documents be more accessible to all clinicians through MEDLINE and PubMed and that radiologists be familiar with accessing and implementing these Practice Parameters. The PPs are high-quality documents reflecting the vast expertise of imaging specialists, and both clinicians and patients will benefit from these documents being readily available and identifiable when undertaking medical literature searches.

We suggest that practitioners gain a high degree of familiarity with accessing Practice Parameters. Doing so will provide additional reference and access to the PPs when medical literature searches are undertaken or when questions arise regarding best practices. Such an approach will ensure that future neuroradiology clinical guidelines or technical standards documents are provided as broad an exposure as possible. This effort could enhance the visibility and accessibility of the quality of practice for neuroradiologists, provide needed clinical guidance to practice state-of- the-art neuroradiology/radiology, and ensure the visibility of our valuable contributions to both individual patient care and collective patient outcomes.

Disclosures: John E. Jordan-OTHER RELATIONSHIPS: Past Chair, Committee on Practice Parameters, Neuroradiology, American College of Radiology. Alexander Norbash—UNRELATED: Board Membership: Stryker, GE Healthcare, GE Data, Penumbra, Comments: scientific advisory boards; Stock/Stock Options: Boston Imaging Core Laboratories, Comments: Cofounder.

\section{REFERENCES}

1. Graham R, Mancher M, Miller Wolman D, et al, eds. Institute of Medicine (US) Committee on Standards for Developing Trustworthy Clinical Practice Guidelines. Clinical Practice Guidelines We Can Trust. Washington, DC: National Academies Press; 2011

2. National Institutes of Health: National Center for Complementary and Integrative Health. Clinical Practice Guidelines. http://nccih.nih. gov/health/providers/clinicalpractice.htm. Accessed July 20, 2018

3. Hoesing, H. Joint Commission International. Clinical Practice Guidelines. Closing the gap between theory and practice. Elsevier, 2016 https://www.elsevier.com/_data/assets/pdf_file/0007/190177/ JCI-Whitepaper_cpgs-closing-the-gap.pdf. Accessed July 23, 2018

4. Omnibus Budget Reconciliation Act of 1989, 42 U.S.C. S 901, Public Law 101-239. (1989)

5. Healthcare Research and Quality Act of 1999, Title IX, 42 U.S.C. S 901

6. Medicare Improvements for Patients and Providers Act, 42 U.S.C. S 304, Public Law 110-275 (2008)

7. Manchikanti, L, Falco, Singh V, et al. An update of comprehensive evidence-based guidelines for interventional techniques in chronic spinal pain, Part I: introduction and general considerations. Pain Physician 2013;16:S1-48 Medline

8. Guiberteau MJ, Wilcox PA, Bjork S. Practice guidelines: the radiology perspective. J Am Coll Radiol 2004;1:92-97 Medline

9. American College of Radiology. Practice Parameters and Technical Standards: Development and Revision Handbook. http://www.acr. org/-/media/ACR/Files/Practice-Parameters/DevelopmentHandbook. pdf. Accessed July 16, 2018

10. American College of Radiology. Practice Parameters and Technical Standards. https://www.acr.org/Clinical-Resources/PracticeParameters-and-Technical-Standards. Accessed July 12, 2018

11. Wintermark M, Colen $\mathrm{R}$, Whitlow $\mathrm{CT}$, et al. The vast potential and bright future of neuroimaging. $\mathrm{Br} J$ Radiol 2018;91:20170505 CrossRef Medline

12. Porter ME. What is value in health care? $N$ Engl J Med 2010;363: 2477-81 CrossRef Medline

13. Narayan A, Cinelli C, Carrino JA, et al. Quality measurements in radiology: a systematic review of the literature and survey of radiology benefit management groups. J Am College Radiol 2015;12: 1173-81 CrossRef Medline

14. Hubbard IJ, Harris D, Kilkenny MF, et al. Adherence to clinical guidelines improves patient outcomes in Australian audit of stroke rehabilitation practice. Arch Phys Med Rehabil 2012;93:965-71 CrossRef Medline

15. Gagliardi AR, Brouwers MC. Integrating guideline development and implementation: analysis of guideline development manual instructions for generating implementation advice. Implement Sci 2012;7:67 CrossRef Medline

16. AHRQ (Agency for Healthcare Research and Quality). Saving Lives and Saving Money: Hospital-Acquired Conditions Update. Washington, DC, 2015. https://www.ahrq.gov/professionals/quality-patientsafety/pfp/interimhacrate2014.html. Accessed July 20, 2018

17. List of All Journals Cited in PubMed. NIH: National Library of Medicine. http://www.nlm.nih.gov/bsd/serfile_addedinfo.html. Accessed July 20, 2018 